http://jmscr.igmpublication.org/home/ ISSN (e)-2347-176x ISSN (p) 2455-0450 crossref DOI: https://dx.doi.org/10.18535/jmscr/v8i2.31

\title{
Overdiagnosis of drug Resistant EPTB (Thyroid)
}

\author{
Authors \\ Nirmal Chandra Satpathy ${ }^{1}$, Pravati Dutta ${ }^{2}$, Rekha Manjhi ${ }^{3}$, Sudarsan Pothal ${ }^{4}$, \\ Aurobindo Behera ${ }^{5}$, Gourahari Pradhan ${ }^{6}$ \\ ${ }^{1}$ Post Graduate, ${ }^{2}$ Professor and HOD,${ }^{3,4}$ Associate Professor,${ }^{5,6}$ Assistant Professor \\ Department of Pulmonary Medicine, VSS institute of Medical Science and Research \\ Burla, 768017, India
}

\begin{abstract}
Introduction: Tuberculosis of thyroid gland is extremely uncommon. The incidence is low even in countries where the prevalence of tuberculosis is high. Objective of this case report is to present a case of over-diagnosed thyroid tuberculosis and to discuss its clinical presentation, investigations, observations and management.
\end{abstract}

Case Presentation: A 38-year old female presented to Pulmonary Medicine department (Tertiary care hospital) with a 6months history of slowly progressive neck swelling, weakness and loss of appetite. On examination there was a firm swelling over neck of size $3 \mathrm{~cm} \times 4 \mathrm{~cm}$. There was no previous history of tuberculosis.

Investigation: Chest radiograph showed no abnormality, sputum smear was found negative for AFB, Mantoux test with $5 T U$ revealed an in duration of $12 \mathrm{~mm}$. Thyroid profile was normal. FNAC of thyroid swelling was suggesting granulomatous thyroiditis. Biopsy of thyroid swelling was suggesting autoimmune thyroiditis. CBNAAT of Biopsy specimen of Thyroid revealed MTB detected and Rifampicin resistant. At the same time liquid culture of that biopsy specimen of thyroid was done and later found to be negative.

Observation: The case was found to be over diagnosed as DR Thyroid TB. This case was referred to DR$T B$ Centre VIMSAR for initiation of DR-TB Regimen. But no ATT was given and kept under observation. Follow-up examination after two months showed resolutions of signs and symptoms.

Conclusion: Thyroid tuberculosis is rare form of tuberculosis. So patient not exposed to TB or any contact history of DR-TB patient should be judiciously treated to prevent overdiagnosis.

Keywords: Thyroid tuberculosis, Extra-pulmonary Tuberculosis, CBNAAT.

\section{Introduction}

Tuberculosis of the thyroid gland is a rare clinical entity ${ }^{1}$. It is a rare disease even in the countries where tuberculosis is endemic. Compared to pulmonary tuberculosis, extrapulmonary tuberculosis may have different clinical manifestations and may be difficult to diagnose. For accurate diagnosis, clinical and radiological features are nonspecific and histological examination is required ${ }^{2}$. The diagnosis is often difficult as the clinical presentation has no distinct characteristics. But sometimes over diagnosis may occur.

\section{Case Report}

A 38-year old female housewife with no addiction presented to Pulmonary Medicine department, VIMSAR, Burla with a 6 month history of slowly 


\section{JMSCR VoI||08||Issue||02||Page 155-157||February}

progressive neck swelling, weakness and loss of appetite. There was no complaint of cough, dysphagia, dysnoea and hoarseness of voice. On examination there was a firm swelling over neck of size $3 \mathrm{~cm} \times 4 \mathrm{~cm}$, which moves with deglutition, non tender without sign of inflammation. There was no previous history of tuberculosis or any contact history with any DR-TB patient.

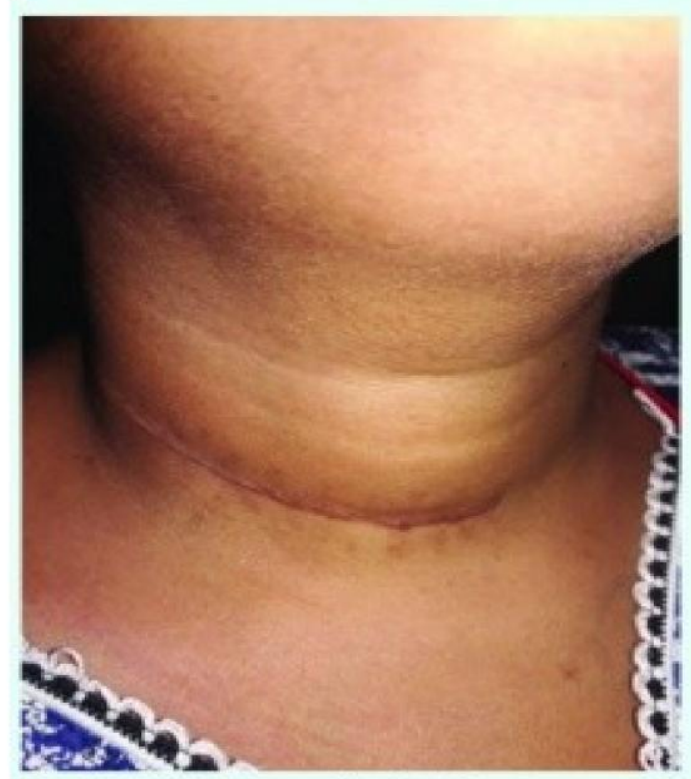

Recent Photograph showing scar mark

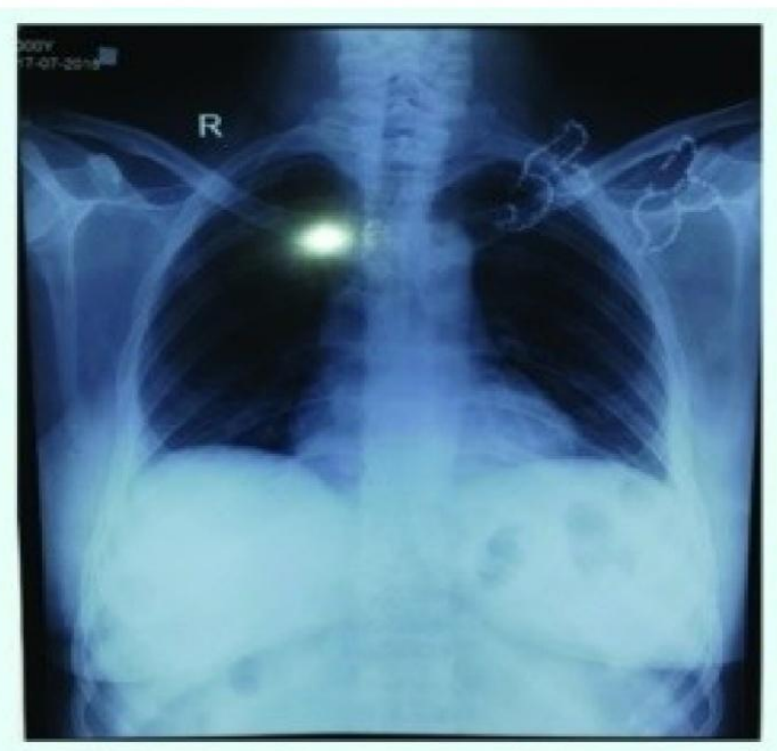

Normal Chest X-Ray (PA view)

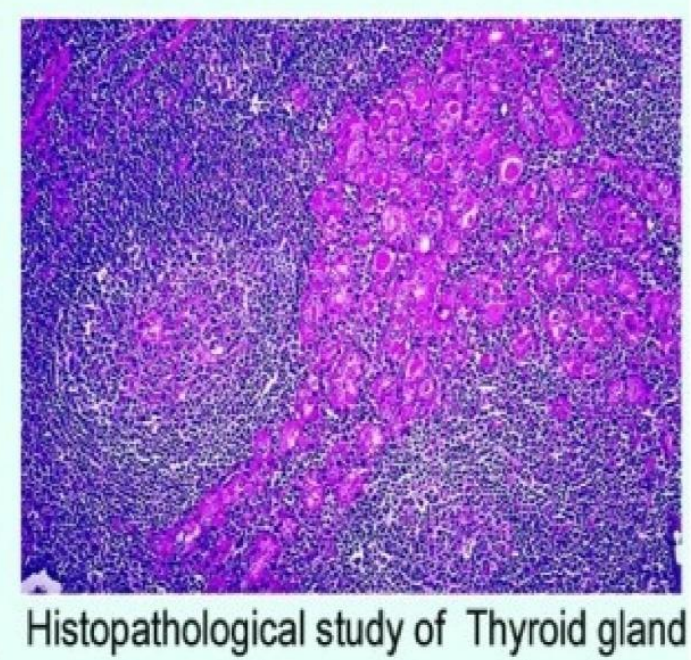

Investigation

Chest radiograph showed no abnormality. Sputum smear for AFB was found negative. Mantoux test with 5TU revealed an in duration of $12 \mathrm{~mm}$. Thyroid profile was normal. Complete blood hemogram was normal. Blood glucose and renal function test were normal. HIV, HBV and $\mathrm{HCV}$ were non-reactive.USG of Neck: showed bulky right lobe of thyroid with a cystic nodule. A lymph node of size $5 \mathrm{~mm}$ noted adjacent to right lobe of thyroid. FNAC of Thyroid swelling: showed clumps of benign follicular cells, few intact follicle, lymphocyte plasma cells, giant cell, clumps of epithelioid cells, numerous cyst macrophage and haemosiderin laden macrophage. There were also blotches of colloid seen, suggesting granulomatous thyroiditis. Histopathological study of thyroid swelling showed colloid filled thyroid follicle mixed with multiple lymphoid follicles, many of which show germinal centre along with hurthle cell and evidence of folliculolysis. Suggesting Autoimmune thyroiditis, Florid type. CBNAAT of Biopsy specimen of Thyroid revealed MTB detected and Rifampicin resistant. At the same time liquid culture of that biopsy specimen of thyroid was done and later found to be negative.

\section{Discussion}

Tuberculosis of the thyroid gland is an extremely rare disease. According to the literature, the frequency of thyroid tuberculosis is $0.1 \%-0.4 \%^{3}$. 
Das et al. reported $0.6 \%$ prevalence of tuberculosis among 1283 thyroid lesions. The diagnosis of thyroid tuberculosis is difficult even in the modern era because it can present in many different ways ${ }^{3}$.

This case was found to be over-diagnose as Rifampicin resistant (RR) Thyroid TB. This case was referred to DR-TB Centre VIMSAR for initiation of DR-TB Regimen. But no ATT was given and kept under strict observation and follow up. She was advised to attend Pulmonary Medicine department, VIMSAR, Burla if she notices any changes in thyroid swelling and/or any respiratory symptoms appears. Follow-up examination after two months showed resolutions of signs and symptoms.

When culture is negative and GeneXpert is positive, it had been seen that the result of Gene Xpert was very low or low positive. PCR test amplifies any DNA, of live or dead bacilli. Therefore, while diagnosing a person with active extrapulmonary tuberculosis clinicians need to be very cautious using it as sole method. Clear history of treatment with ATT is required to avoid false positive result. ${ }^{4,5}$

\section{Conclusion}

Nowadays there is an increasing trend of drug resistance in EPTB but many are overdiagnosed. In Drug resistant EPTB we should wait for liquid culture rather starting DR-TB Regimen by observing CBNAAT report. Thyroid tuberculosis is rare form of tuberculosis. So patient not exposed to TB or any contact history of DR-TB patient overdiagnosed as DR-EPTB should be judiciously treated to avoid unnecessary exposure to 24 months of DR-TB regimen. Drug resistance EPTB is often a diagnostic challenge to treating physician. So strong clinical suspicion, judicious interpretation of diagnostic modalities helps in prevention of overdiagnosis.

\section{References}

1. Mondal A and Patra D K, "Efficacy of fine needle aspiration cytology in the diagnosis of tuberculosis of thyroid gland" a study of 18 cases," Journal of Laryngology and Otology, vol. 109, no.1, pp. 36-38, 1995.

2. F. W. Rankin and A. S. Graham, "Tuberculosis of the thyroid gland," Annuals of Surgery, vol. 96, no. 4, pp. 625-648, 1932.

3. Das DK, Pant CS, Chachra KL, Gupta AK. Fine needle aspiration cytology diagnosis of tuberculous thyroiditis: a report of eight cases. Acta Cytol. 1992;36:517-522.

4. Van Rie A, Page-Shipp L, Scott L, Sanne !,Stevens W. Xpert (R) MTB/RIF for point-of-care diagnosis of TB IN HighHIV burden, resource-1 imited countries: hype or hope? Expert Rev Mal Diagn. 2010; 10(7):937-46.

5. Barnard DA, lrusen EM, Bruwer JW, Plekker D, Whitelaw AC, Deetlefs JD, et al. Koegelenberg The utility of Xpert MTB/RIF performed on bronchial washings obtained in patients with suspected pulmonary tuberculosis in a high prevalence setting. BMC Pulm Med. 2015; 15:103. 